\title{
Clés de lecture piétonne des espaces publics aménagés de tramway : l'influence de l'environnement sensible sur les comportements de traversée
}

\author{
How the pedestrian perceive urban spaces with tramway: \\ the influence of the urban environment on the crossing behavior
}

\author{
Élis Maître · Marine Millot \\ Reçu le $1^{\text {er }}$ décembre 2012 ; accepté le 21 octobre 2013 \\ (C) IFSTTAR et Éditions NecPlus 2013
}

\begin{abstract}
Résumé Dès les années 1980 un redéploiement des réseaux de tramways s'effectue au cœur des villes françaises. Dans un contexte de promotion à l'échelle de l'urbain d'objectifs de développement durable et de compétition entre villes, le tramway (en tant qu'outil des politiques de transport) devient aussi un outil de projet urbain pour redistribuer l'espace. Les aménagements de tramway créent ainsi de nouvelles formes d'espaces publics en recomposant l'ensemble de l'espace viaire entre les façades. La littérature internationale met notamment en évidence des difficultés, en termes de conditions d'usage et de sécurité, au sein de ces nouveaux espaces. Les piétons étant les usagers les plus vulnérables, leur représentation de ces espaces publics aménagés de tramway est à considérer puisque de celle-ci dépend leur sécurité. Le travail présenté ici définit des clés de lecture piétonne des espaces publics aménagés de tramway à travers l'analyse des formes construites, des sens et des pratiques. La méthode construite permet de proposer un outil d'analyse des choix effectués par les piétons pour leurs trajets et des traversées d'aménagement de tramway.
\end{abstract}

Nous tenons à remercier Madame Frédérique Hernandez et Madame Brigitte Bertoncello pour leur suivi ainsi que leurs conseils. Nous remercions également les spécialistes du service ARTU (Aménagement réseaux transports urbains) du Centre d'études techniques de l'équipement Méditerranée pour leur coopération à ce travail.

Élis Maître $(\square)$

CETE Méditerranée, DAT-ARTU, Pôle d'activités CS 70499

13593 Aix-en-Provence

e-mail : elisa.maitre@gmail.com

Marine Millot $(\square)$

CETE Méditerranée, DAT-ARTU, Pôle d'activités CS 70499

13593 Aix-en-Provence

e-mail : marine.millot@developpement-durable.gouv.fr
Mots clés piéton · perception · tramway $\cdot$ environnement urbain $\cdot$ ambiance $\cdot$ comportement $\cdot$ traversée des voies

Abstract Since the eighties, tramway networks have expanded in the center of French towns. The context is marked by promotion of urban area, sustainable development and competition between cities. And more than a tool of transport policy, the tramway becomes a tool to revitalize city centers and better share the space. The tramway layout involves new design of public spaces between façades. The international literature highlights difficulties in term of conditions of use and safety in these new spaces. Pedestrians are the most vulnerable users. Their perception of these spaces must be analyzed to improve their safety. This study defines tools to know how pedestrians perceive urban spaces with tramway. It is based on the analysis of built form, senses and uses. The developed method enables to analyze the choices made by pedestrians for their route and the crossings of tramway lanes.

Keywords Pedestrian · Perception · Tramway · Urban environment · Atmosphere - Behaviour · Pedestrian crossings of tramway lines

\section{Contexte et problématique}

Dès les années 1980 en France, dans un contexte de compétitivité entre villes, de recherche d'alternative à l'emprise spatiale de l'automobile puis de promotion du développement durable, un regain d'intérêt se manifeste pour les systèmes de transports guidés [1]. De nombreuses villes françaises vont ainsi réintroduire le tramway au cœur des centres urbains, en commençant par Nantes en 1985. En 1988, la ville de Grenoble associe clairement la mise en place du tramway avec un projet de requalification urbaine. Le tramway, en tant que mode de transport, devient aussi 
un outil de projet urbain pour revitaliser les centres-villes et redistribuer l'espace [2]. La mise en place de réseau de tramway est ainsi un vecteur d'importantes mutations des territoires urbains, qu'il s'agisse de leur organisation spatiale ou de la morphologie des tissus traversés [3].

Ces nouvelles formes d'espaces publics générées par la mise en place de tramways soulèvent des questions en termes de lisibilité pour les usagers piétons. À grande échelle, le réseau de tramway semble participer à l'amélioration de la lisibilité de la ville au sens de K. Lynch, c'est-à-dire la faculté de se repérer dans l'espace de la ville, notamment en s'orientant par rapport à des points de référence urbains (bâtiments emblématiques, lieux bien fréquentés, etc.). L'image qu'ont les piétons de leur environnement urbain, ou autrement dit la représentation qu'ils s'en font, participe à leur capacité à s'orienter, à leur facilité à se repérer et à pouvoir se diriger dans leur environnement urbain [4].

Cependant, à l'échelle de l'espace public, le tramway semble complexifier la lecture et la compréhension des aménagements urbains. En effet, l'insertion du tramway en France redessine et recompose l'ensemble de l'espace public entre les façades. Les aménagements de tramway (éléments de l'espace public et de la voirie qui constituent la ligne de tramway) multiplient le nombre ainsi que le type de voies à traverser et introduisent une priorité absolue en intersection. Ils peuvent être soit partagés avec le reste de la circulation, soit en site propre avec une plateforme en position latérale, bilatérale, axiale, différenciée ou non par des bordures et revêtements de sol. La réintroduction des réseaux de tramway au sein d'un espace viaire construit autour de l'automobile engendre ainsi une nouvelle complexité, avec notamment des effets de coupure, nécessitant la compréhension par les usagers de ces espaces et en particulier les piétons $[5,6,7]$.

Ce manque de lisibilité joue un rôle particulièrement important dans les processus d'accident des piétons. La littérature internationale a montré que cela pouvait engendrer des prises d'informations inappropriées, en particulier aux niveaux des intersections avec le tramway $[8,9]$, des incompréhensions de la configuration des lieux ou des dépassements des capacités cognitives [10,11,12].

$\mathrm{La}$ réintroduction des réseaux de tramway soulève ainsi la question de la compréhension de ces nouvelles formes d'espaces publics par les usagers piétons. Selon le modèle de l'opérateur humain issu de la psychologie, la compréhension des piétons de leur environnement urbain, puis leur prise de décision et l'exécution de l'action, passe en premier lieu par la perception (au sens de l'organisation), puis par l'interprétation de leur sensation (en les complétant par des images ou des souvenirs) [13]. La représentation piétonne des environnements urbains est à considérer, puisque de celle-ci dépendent la sécurité, la présence humaine dans la rue, la sociabilité, l'urbanité, l'appropriation de l'espace et la conscience urbaine. C'est la raison pour laquelle cet article traite des questions suivantes : comment les espaces publics accueillant des aménagements de tramway sont-ils perçus par les piétons ? En particulier, quelle influence ont ces environnements reconfigurés par les tramways sur le comportement des piétons en traversée (zone de conflit potentiel entre ces différents modes) ? Pour traiter de ces questions il convient d'avoir à l'esprit l'idée que le piéton n'est pas un usager de l'espace public comme les autres car il établit des interactions complexes avec son environnement urbain [14]. L'environnement urbain est la synthèse du cadre physique composé des objets qui forment le dispositif spatial et de l'ensemble des relations établies par les individus dans cet espace [15]. En ce sens, le piéton est constitutif de son environnement urbain, il s'y projette et agit avec lui.

$\mathrm{Ce}$ travail amène à interroger l'importance de la lecture piétonne de l'environnement. Selon Chelkoff et Thibaud [16], l'environnement au sens large est constitutif d'une ambiance impliquant les pratiques collectives du lieu-mouvement. L'interrelation avec l'environnement a une incidence sur le déplacement des piétons et particulièrement sur l'expressivité de leur corps et leur mode d'orientation perceptive [17]. L'œuvre de Goffman [18] met en évidence le fait que les piétons actualisent l'ensemble de leurs compétences perceptives et expressives en fonction des caractéristiques de leur environnement urbain. Les piétons entretiennent ainsi une « relation affective » à leur environnement urbain [19]. Thomas [20] dans son étude sur les trajectoires de l'accessibilité, établit une typologie des manières de cheminer des piétons au sein de l'espace public urbain en fonction de six types de milieux ambiants. Lors de la déambulation en milieu ambiant «tempéré » et du noctambulisme en milieu ambiant «attractif », les piétons sont en adéquation avec leur environnement. La traversée en milieu ambiant « ambigu » et le piétinement en milieu ambiant «saturé » rendent compte d'un rapport problématique. Le passage en milieu ambiant « transitoire » et la fuite des piétons en milieu ambiant « délaissé » attestent d'une relation purement fonctionnelle à leur environnement. Chaque variation de l'environnement urbain a un impact sur la qualité du cheminement piéton, sur les pratiques sociales et sur l'ordre du sensible [21]. De plus, la relation des piétons à leur environnement sensible s'analyse par une hétérogénéité des modes d'attention [22]. L'importance du rôle des sens dans l'interaction des piétons avec leur environnement révèle ainsi la complexité du sujet traité. L'environnement sensible des aménagements de tramway mérite une attention particulière car celui-ci semble influencer l'allure de déplacement, la conduite perceptive, la position et l'orientation des piétons. 
Concernant la question de la traversée de voie, zone de conflit prioritaire avec le tramway, le recours aux analyses des comportements est un enjeu majeur en termes de sécurité des piétons. La tâche de traversée de voie, notamment en intersection, est complexe et fait appel aux processus perceptifs et cognitifs des piétons [14]. Les processus perceptifs des piétons prennent notamment en compte leur acuité visuelle et leur audition. Les processus cognitifs permettent aux piétons la prise de décision et le traitement de l'information de leur environnement. De plus, la notion de prise de risque des piétons est fondamentale pour l'analyse de la traversée de voie [23]. Elle intervient lorsque les piétons veulent raccourcir leurs distances de traversée et leur temps d'attente [24], comme variable à ajuster entre la réponse aux besoins de mobilité et l'évitement des situations d'accidents.

Les piétons évaluent le risque selon leurs perceptions de l'environnement physique, leurs représentations internes et leurs motivations. En effet, les éléments de l'environnement peuvent avoir un impact sur la pénibilité et l'éventuelle transgression de la réglementation. Enfin, les piétons considèrent la traversée comme une tâche secondaire par rapport à leur objectif [25]. Selon que les piétons soient pressés ou qu'ils se promènent, ils réévaluent leur appréciation du risque.

L'étude de la perception piétonne des aménagements de tramway est essentielle pour une meilleure compréhension des usages et pratiques de ses nouveaux espaces. Cependant, comme le souligne Stransky [26], l'influence de l'infrastructure en tant que support du déplacement et de son environnement sensible sur l'attractivité des piétons, est très peu détaillée ; les données sont rares, souvent incomplètes et indisponibles. Ainsi, à travers une analyse des formes construites, des usages et des sens, il s'agit dans cet article de se poser la question de la perception piétonne des aménagements de tramway. L'hypothèse majeure est que des éléments de l'environnement urbain des aménagements de tramway, de l'ordre physique (morphologie urbaine), social (relations entre les individus, usages) ainsi que sensible (ambiances) exercent une influence sur les comportements des piétons dans leurs déplacements dans l'espace public et plus particulièrement en traversée de voie.

Afin de traiter la question de la perception piétonne des aménagements de tramway pour en déterminer leur compréhension, notamment en traversée, une méthode est construite en repositionnant la question à différentes échelles. Les piétons composent avec leur environnement : leurs déplacements impliquent une réflexion portant à la fois sur l'échelle de l'espace public et de la traversée de voie. L'étude d'un objet dans son milieu ne peut être dissociée d'une réflexion sur les relations entre les parties et le tout, et réciproquement. Différentes échelles interviennent ainsi, de l'étude des axes majeurs intégrant des aménagements de tramway pour identifier les zones de conflits potentiels, jusqu'à l'analyse d'une trame viaire spécifique où le zoom le plus précis est réalisé au niveau de la traversée de voie.

Dans le travail proposé ici, la méthode comporte trois étapes. En premier lieu, une analyse séquentielle est réalisée à partir d'éléments de diagnostic des espaces publics aménagés de tramway. Elle permet de mettre en évidence des séquences spécifiques à approfondir où le niveau de conflit potentiel peut être important. Une séquence se définit comme un morceau d'environnement homogène du point de vue de l'expérience vécue. En second lieu, une analyse sensorielle permet de définir les différents milieux ambiants de ces espaces publics et leur influence sur les façons dont les piétons cheminent. En troisième lieu, une plongée dans l'univers des pratiques est effectuée à l'échelle de la traversée des aménagements de tramway. La deuxième et la troisième étape de la méthode sont ensuite mises en corrélation pour analyser l'influence de l'environnement sensible sur la perception piétonne des aménagements de tramway et sur les comportements de traversée. Pour tester l'applicabilité de cette méthode, chaque étape a été déployée sur la ligne 2 de tramway de la commune de Marseille.

\section{À l'échelle de la ligne : des éléments de diagnostic des aménagements de tramway}

La première étape de la méthode traite de la qualité des aménagements de tramway en tant que support des déplacements de piétons. La méthode employée s'appuie sur des "descriptions en marche », consistant à observer une situation « naturelle» de terrain, pour ensuite établir un corpus, le plus complet possible, en réalisant des relevés systématiques [27]. L'analyse séquentielle présentée ici, met en évidence une réflexion sur la construction de nouveaux critères permettant de relever les qualités et les dysfonctionnements des aménagements de tramway du point de vue des piétons. L'observation n'est pas «participante » mais reste discrète. Cette analyse séquentielle, au-delà d'un repérage précis, est un outil d'analyse des rapports entre les piétons et leur environnement.

\section{L'analyse séquentielle des tronçons de la ligne de tramway}

Il s'agit de caractériser l'environnement de tramway, pris dans ses dimensions urbaines et saisies dans ses paramètres influant sur la perception sensorielle des piétons. Une grille d'analyse est ainsi construite (Fig. 1) afin 


\begin{tabular}{|c|c|}
\hline Centralité & Centre urbain - première couronne - périphérie \\
\hline Forme urbaine & Densité, hauteur, positionnement du bâti \\
\hline Capacité à attirer & $\begin{array}{l}\text { Pôles générateurs de déplacements } \\
\text { Mixité des fonctions }\end{array}$ \\
\hline Fonctionnalité & $\begin{array}{l}\text { Séparation ou mixité des flux tramways et voitures } \\
\text { Positionnement de la plateforme de tramway } \\
\text { Volume de trafic routier et vitesses } \\
\text { Nombres des voies de circulation } \\
\text { Sens de circulation } \\
\text { Correspondances possibles }\end{array}$ \\
\hline Confort de l'espace public & $\begin{array}{l}\quad \text { - Le long de la voie : } \\
\text { Confort du cheminement } \\
\text { Largeur des trottoirs } \\
\text { Densité des piétons } \\
\text { Espaces d'arrêt (éléments d'hospitalité de l'espace public) } \\
\text { Distinction de la plateforme } \\
\quad \text { - En traversée : } \\
\text { Traversées piétonnes adaptées } \\
\text { Le piéton se sent-il canalisé/libre de traverser? }\end{array}$ \\
\hline Perception sensorielle & $\begin{array}{l}\text { Propreté des lieux } \\
\text { Esthétique visuelle } \\
\text { Odeurs/bruits } \\
\text { Sentiment d'aération/oppression des volumes bâtis } \\
\text { Perspective paysagère } \\
\text { Monotonie ou variété des paysages } \\
\text { Sentiment de sécurité } \\
\text { Sensation de bien-être/mal-être et de calme }\end{array}$ \\
\hline
\end{tabular}

Fig. 1 Grille d'analyse par tronçon du niveau de conflit potentiel dans l'interaction piéton-tramway [28]

de cerner toute la complexité des comportements des piétons et de leurs interactions avec l'environnement. La première approche prend en compte la centralité, la forme urbaine, la capacité à attirer des piétons avec notamment la présence de pôles générateurs de déplacement. Cette approche évalue également les éléments de confort de l'espace public pour les piétons et la fonctionnalité de l'infrastructure (tel que le positionnement de la plateforme de tramway). La seconde approche considère les éléments de la sensorialité du lieu influençant sur la perception des piétons.

L'élaboration de cette grille d'analyse optimise la récolte, la présentation et l'évaluation des données de l'environnement piéton des aménagements de tramway. L'analyse séquentielle des tronçons est réalisée à partir de quatre critères majeurs : la capacité à attirer les piétons (pôles générateurs de déplacement, mixités des fonctions), la fonctionnalité des aménagements (positionnement de la plateforme, volume de trafic routier et vitesses), les éléments de confort mis à disposition (espaces d'arrêt, largeur des trottoirs) et le sentiment de bien-être (propreté des lieux, bruits). L'analyse de ces quatre critères met en évidence la classification suivante, selon l'appréciation du niveau de conflit potentiel du plus faible au plus fort (Fig. 2). Le niveau de conflit est faible quand le tronçon est peu attractif vis-à-vis des piétons ou, au contraire, il est très attractif mais bien adapté en terme d'aménagement, de sentiment de bien-être, etc. Ainsi, cette analyse permet d'identifier les tronçons posant des problèmes de perception et de condition d'usage pour les piétons.

L'objectif est d'approfondir l'étude des tronçons dont les environnements de tramway sont qualifiés d' « ambigus » par les piétons, c'est-à-dire ceux où le niveau de conflit potentiel est important. En effet, ces séquences spécifiques sont susceptibles d'être les plus problématiques du fait d'un paradoxe entre une forte présence de piétons et des dysfonctionnements impactant sur la perception des aménagements de tramway. Selon Levy, «le voyageur (piéton) est peut-être myope, mais c'est lui l'usager, et son errance, ses difficultés quotidiennes dans ces grands espaces publics sont un symptôme de dysfonctionnement et la marque d'une accessibilité parfois insuffisante » [29]. 


\begin{tabular}{|l|l|l|}
\hline $\begin{array}{l}\text { Niveau de conflit } \\
\text { potentiel }\end{array}$ & $\begin{array}{l}\text { Qualité du } \\
\text { cheminement } \\
\text { piéton }\end{array}$ & Analyse séquentielle des tronçons \\
\hline FAIBLE & MÉDIOCRE & $\begin{array}{l}\text { Pas d'attrait de piéton, aucun pôle générateur de déplacement } \\
\text { Le cheminement du piéton est canalisé par les aménagements } \\
\text { Aucun élément de confort mis à disposition } \\
\text { Le piéton ne se sent pas à sa place } \\
\text { Le piéton subit des expériences sensorielles négatives }\end{array}$ \\
\cline { 2 - 3 } & MOYENNE & $\begin{array}{l}\text { Peu d'attrait de piéton } \\
\text { Aménagements peu fonctionnels } \\
\text { Peu d'éléments de confort pour le piéton } \\
\text { Sentiment de bien-être, calme }\end{array}$ \\
\cline { 2 - 3 } & ADAPTÉE & $\begin{array}{l}\text { Attrayant, commerce en rez-de-chaussée } \\
\text { Niveau de trafic routier faible } \\
\text { Éléments de confort mis à disposition } \\
\text { Sentiment de bien-être, agréable pour le piéton }\end{array}$ \\
\hline FORT & AMBIGUË & $\begin{array}{l}\text { Important attrait de piéton, nombreux pôles générateurs de déplacement } \\
\text { Aménagements peu lisibles et peu confortables } \\
\text { Le piéton se sent à sa place et libre de traverser }\end{array}$ \\
\hline
\end{tabular}

Fig. 2 Évaluation du niveau de conflit potentiel de l'environnement de tramway du point de vue du piéton [28]

\section{Des séquences « ambiguës » en termes d'environnement de tramway}

Appliquée à la ligne 2 de tramway de Marseille, la méthode permet d'identifier neuf séquences (Fig. 3). Quatre séquences se distinguent par leur qualification du niveau de conflit fort : le boulevard Longchamp (station Longchamp/Réformés), le cours Belsunce (station Belsunce), la Canebière (station Garibaldi) et la rue République (stations Sadi-Carnot/Joliette).

Ces séquences situées au cœur du centre urbain présentent une densité de piéton élevée avec de nombreux pôles générateurs de déplacement. Les piétons se sentent à leur place et libres de traverser. Cependant, ces séquences ne sont pas toujours fonctionnelles, ni confortables pour les déplacements des piétons. De plus, la diversité d'informations est peu assimilable et lisible par les piétons. L'analyse de ces séquences nécessite ainsi d'être particulièrement approfondie. Par ailleurs, ces séquences spécifiques permettent des comparaisons significatives (Fig. 4, 5).

Les séquences du boulevard Longchamp et du cours Belsunce se caractérisent par des aménagements de tramway traversant une aire piétonne et s'insérant au sein d'un environnement urbain radicalement opposé. L'analyse approfondie de ces deux séquences permet de faire ressortir l'influence de l'environnement urbain des aménagements de tramway sur le comportement des piétons en dehors des voiries.

Les séquences de la rue de la République et de la Canebière s'opposent en terme de position des voies de tramway par rapport à la voirie routière (plateforme en position axiale/bilatérale) et s'insèrent au sein d'environnement urbain aux caractéristiques proches. L'analyse approfondie de ces deux autres séquences permet notamment de révéler l'influence des aménagements de tramway sur les comportements des piétons en comparaison avec les voies routières.

Il ressort de cette première étape méthodologique qu'une diversité d'environnements de tramway se distingue à l'échelle d'une même ligne. Les piétons sont ainsi confrontés à cette complexité. Par ailleurs, cette méthode met en évidence une analyse des liens entre les différents lieux et objets, en fonction des déplacements des piétons. En prenant en compte le point de vue des piétons en tant qu'usagers à part entière de l'espace public, cette méthode leur accorde un statut d'acteur situé au cœur de la chaîne des déplacements et menant des interactions privilégiées avec son environnement urbain. De Certeau [30] évoque la puissance des usages des marcheurs qui par leur mouvement, leur présence corporelle et leur liberté d'action « réalisent les lieux ». 


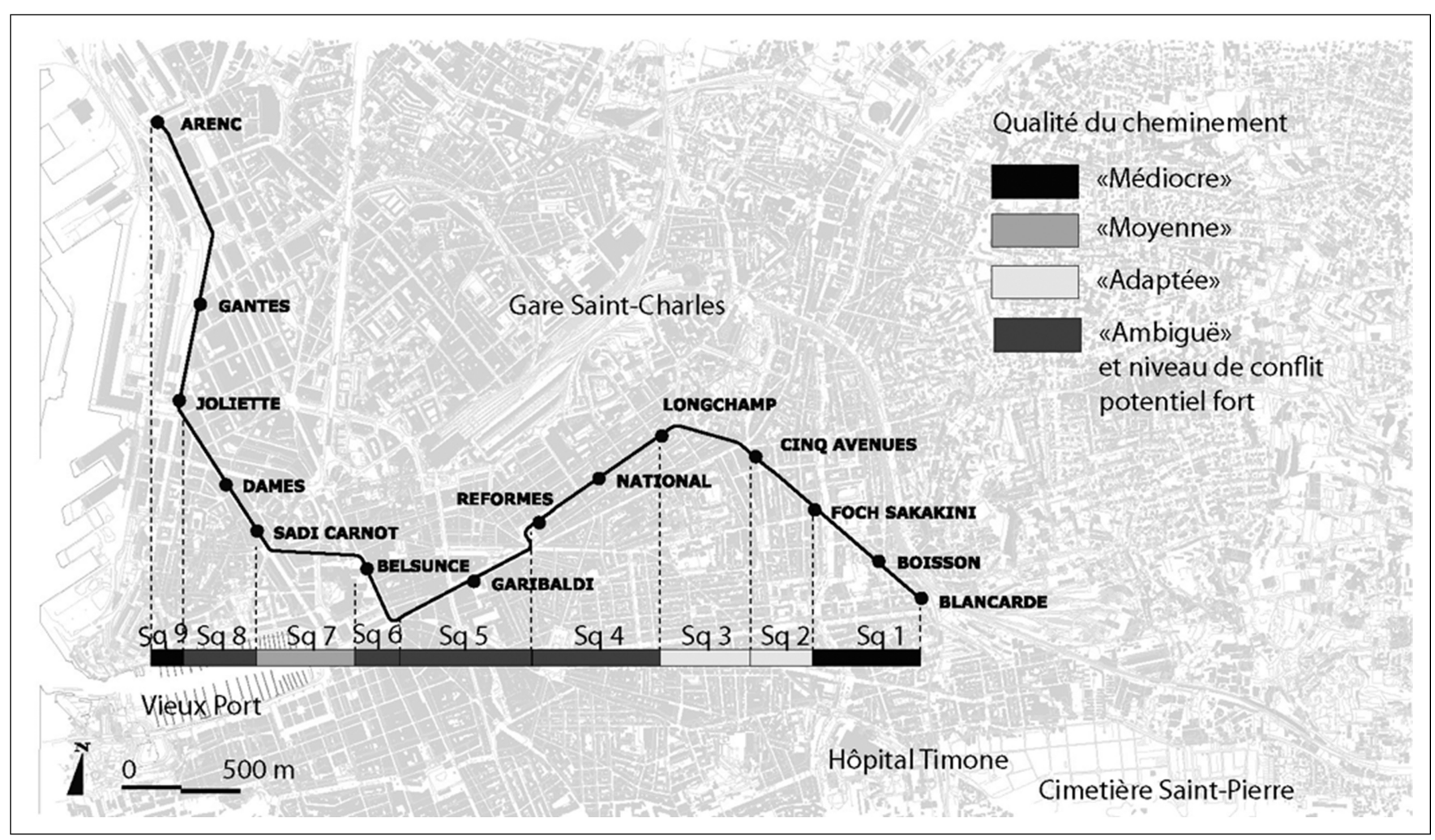

Fig. 3 Environnements de tramway de la ligne 2 de Marseille [28]

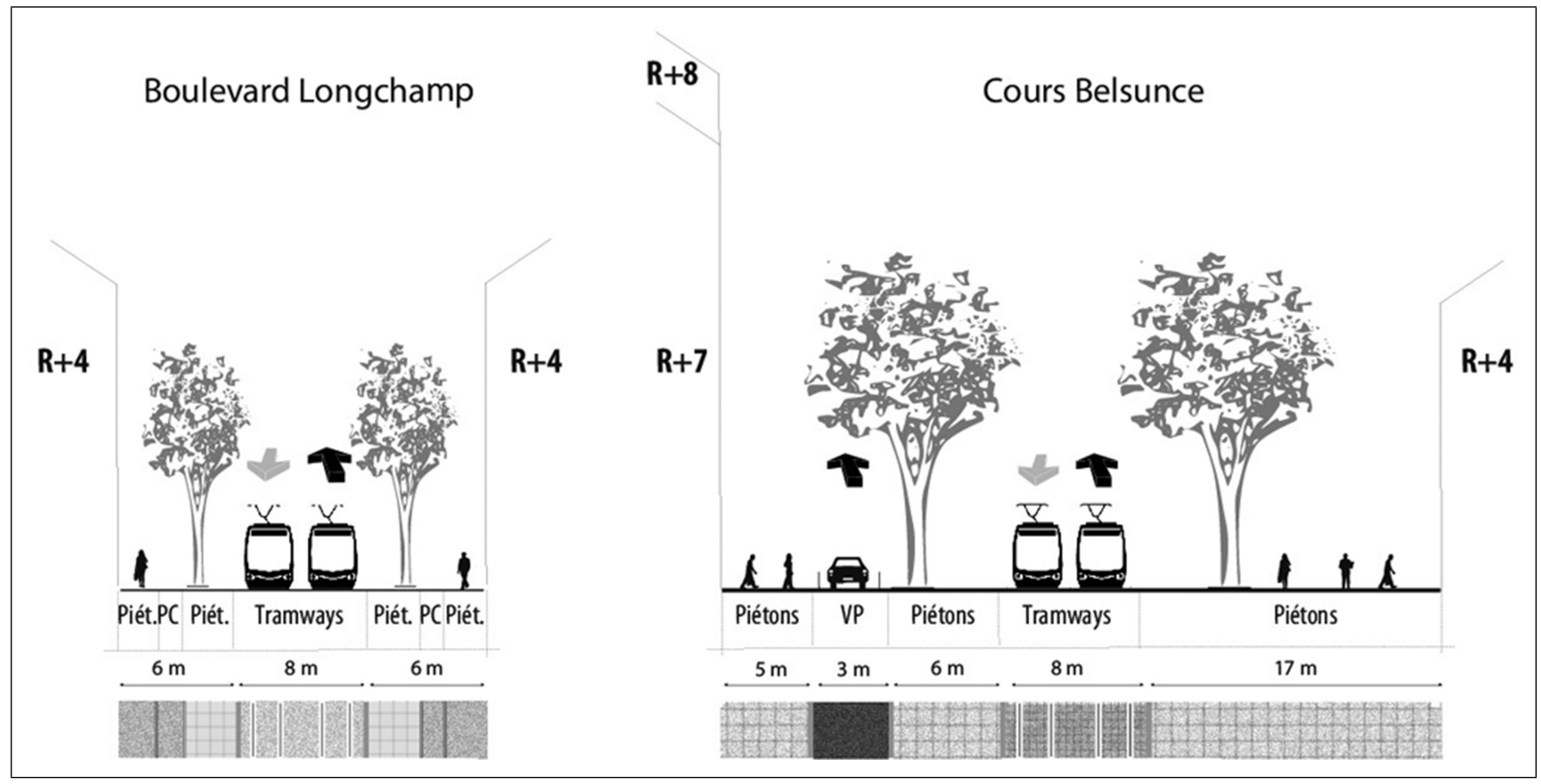

Fig. 4 Coupes transversales du boulevard Longchamp et du cours Belsunce [28]

Necplus 


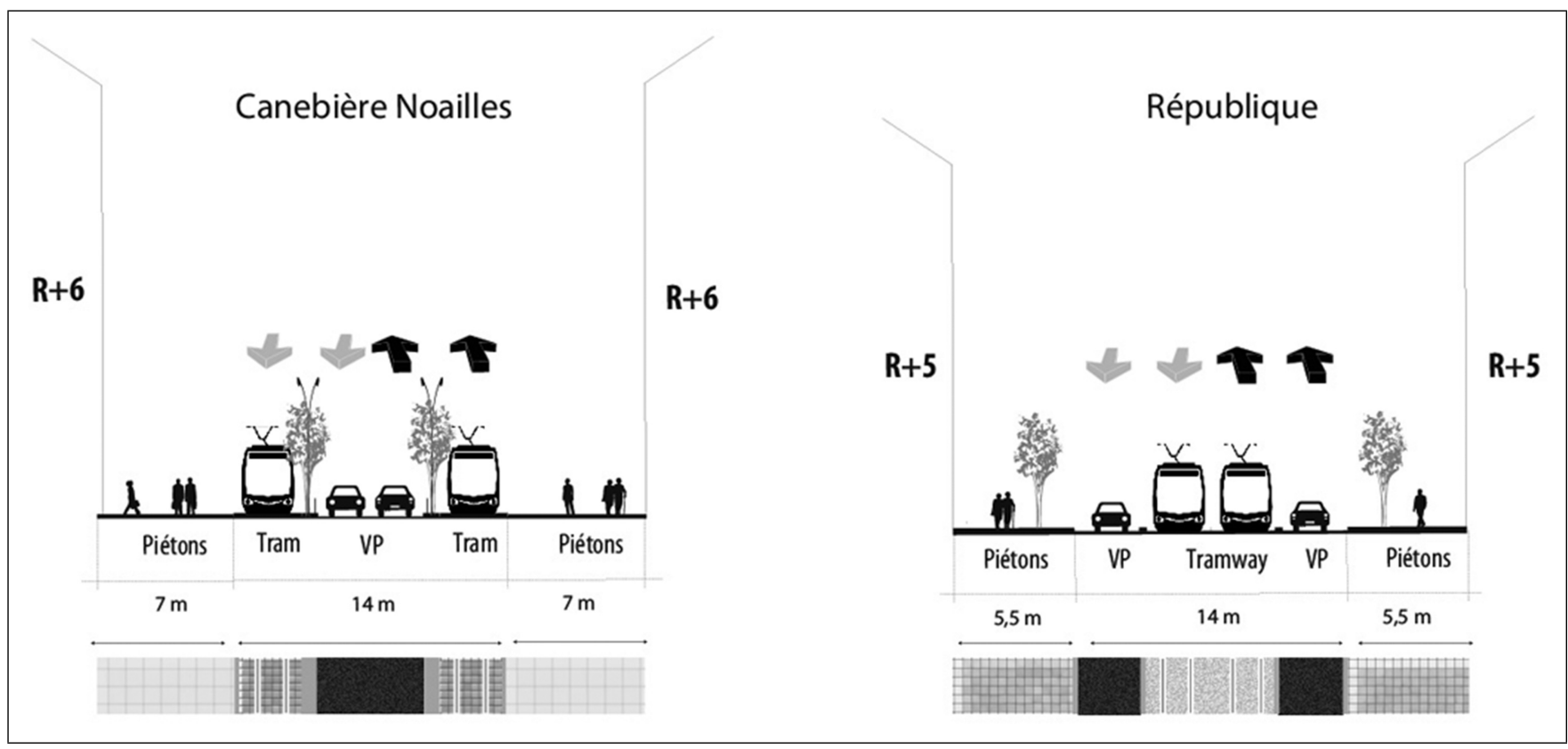

Fig. 5 Coupes transversales de la Canebière et de la rue République [28]

\section{À l'échelle de la séquence : définition des milieux ambiants de tramway}

Certains outils standardisés d'observation des mobilités à l'échelle urbaine existent; tels que les enquêtes ménages, les enquêtes emploi du temps, les enquêtes nationales transport. Ces dispositifs sont cependant peu adaptés à l'appréhension des déplacements piétons dans leur environnement urbain. De plus, les modèles des déplacements piétons sont souvent inspirés des modèles de trafic motorisé et analysent l'interaction entre les acteurs en négligeant le contexte, les contraintes, ainsi que les potentialités perceptives offertes par le site [31]. Ainsi, peu d'études et de données portent sur la façon dont le milieu sensible interfère avec la perception des piétons. La seconde étape de la méthode proposée ici, consiste à étudier les aménagements de tramway par les réactivations sensorielles des piétons à l'échelle des séquences spécifiques à approfondir. La méthode construite s'appuie sur une analyse des formes physiques et des configurations sensibles d'ambiance au niveau du contact des piétons avec leur environnement urbain. Les dimensions sensorielles, morphologiques, sociales et fonctionnelles sont mobilisées.

\section{L'analyse sensorielle}

La méthode permet de déterminer des clés de lecture pour définir l'ambiance des environnements de tramway. Une ambiance urbaine est la synthèse, pour un individu à un moment donné, des perceptions multiples que lui suggère le milieu qui l'entoure [32]. L'ambiance des espaces aménagés de tramway se compose ainsi des facteurs environnementaux perceptibles par les sens et constituent des fragments de l'expérience sensorielle des piétons. La définition du milieu ambiant des aménagements de tramway résulte de la combinaison des différents facteurs sensibles, physiques et sociaux de l'environnement urbain.

Pour définir les ambiances, une grille d'analyse sensorielle des environnements urbains des aménagements de tramway est proposée (Fig. 6). Elle mobilise, tout d'abord, l'ensemble des quatre sens : la vue, l'ouïe, l'odorat et le toucher. Puis elle intègre des éléments d'ordre physique qui font appel à une analyse du cadre bâti. En effet, l'architecture participe notamment à la définition d'ambiances en créant des environnements spécifiques. Les relations spatiales (orientations, expositions, matériaux, volumes et surfaces) sont également étudiées. Enfin, elle intègre les éléments des conduites sociales et de leurs expressivités qui prennent en compte les processus de sociabilité incluant les usages, les actions ainsi que les échanges.

Les piétons actualisent l'ensemble de leurs compétences perceptives et expressives en fonction des caractéristiques du milieu ambiant [18]. De plus, en fonction de l'ambiance de leur environnement urbain les piétons mettent en évidence une relation affective, fonctionnelle, esthétique, induisant une diversité de manière de cheminer [33]. De ce fait, une certaine configuration sensible peut amener des qualités de relations à autrui différentes. La possibilité de 
passage, l'attraction, la répulsion, l'évitement et le détour sont des indicateurs importants des différentes formes d'usages d'un lieu.

Afin d'appréhender ces différents facteurs sensibles, physiques et sociaux des aménagements de tramway, l'analyse est réalisée en quatre étapes. Tout d'abord, l'observation : l'enquêteur, porteur d'un savoir d'expert, se laisse entraîner par la représentation et les aménagements de tramway deviennent une scène. Ensuite, vient l'écoute du rapport entre les sons. Chaque son est identifié (celui de l'animation, de la nature, du confinement, de la résonance), ainsi que le sentiment agréable ou non du son, le rythme et la symbolique qu'il forme. Par la suite, le spectateur devient acteur en s'intégrant aux pratiques et usages du lieu. En effet, une ambiance est indissociable de l'individu qui la perçoit et qui en même temps y contribue par sa présence. Enfin, un retour sur l'expérience est effectué pour organiser les sensations et les transformer en discours, en réalisant un travail de synthèse sous forme de croquis, de récit de voyage, de cartographie, de photographie. À cela est ajoutée une analyse des éléments de la lisibilité des aménagements de tramway pour chacune des séquences spécifiques. Elle permet de définir leurs images du point de vue des piétons usagers de l'espace public.

La synthèse de cette approche permet de qualifier les séquences de tramway et de les rapprocher des six types de milieux ambiants mentionnés précédemment [20]. Le milieu ambiant « tempéré » recèle des vertus reposantes et favorise les rencontres. Le milieu ambiant « attractif » témoigne de qualité divertissante et attractive du piéton. Au sein du milieu ambiant « ambigu », les seuils et limites de l'espace public sont questionnés, le piéton n'est pas en situation d'échange avec autrui, il focalise son attention sur lui-même. Le milieu ambiant « saturé » correspond à un lieu très peuplé (telles que les zones piétonnes ou espaces commerciaux) les sens du piéton sont focalisés sur son action motrice d'évitement d'obstacles. Le milieu ambiant «transitoire», ne participe pas à l'épanouissement du piéton : le milieu sonore et visuel est agressif, défavorable à l'échange et au séjour. Enfin, le milieu ambiant « délaissé » engendre de la crainte pour le piéton.

\section{Des éléments d'ambiances contrastées}

L'« analyse sensorielle » effectuée au sein des quatre séquences spécifiques du terrain d'étude montre des différences d'ambiance perçues par les piétons.

Le boulevard Longchamp évoque « une avenue jardin ».

Le milieu ambiant de la séquence du boulevard Longchamp peut être qualifié de " tempéré ». En effet, en cette journée ensoleillée d'été, la luminosité y est tamisée, elle est filtrée par les platanes et apporte un sentiment de bien-être. Le caractère saisonnier a son importance pour la définition de cette ambiance. De plus, la forme homogène et continue des immeubles apporte un effet de confinement et renforce un effet de visée, c'est-à-dire une mise en perspective mettant en lumière un point de repère important (le palais Longchamp). Le calme règne et une mise entre parenthèses du reste de la vie citadine semble se produire. Seul un effet de réverbération des rires des collégiens vient rompre cette sensation de coupure. L'alignement d'arbres

\begin{tabular}{|c|c|}
\hline Éléments de sensorialité des lieux & $\begin{array}{l}\text { Point de vue } \\
\text { Direction, diffusion de la lumière } \\
\text { Texture, orientation, couleur des surfaces } \\
\text { Effets visuels } \\
\text { Éléments d'histoire des lieux } \\
\text { Agencement des sons (rythmes, intensité) } \\
\text { Propagation des sources de sons } \\
\text { Modalités temporelles de la production des sons }\end{array}$ \\
\hline Éléments de l'ordre physique & $\begin{array}{l}\text { Relations spatiales, orientations, expositions } \\
\text { Matériaux, volumes, surfaces }\end{array}$ \\
\hline Éléments des conduites sociales & $\begin{array}{l}\text { Sentiment d'insécurité } \\
\text { (menaces physiques d'objet en mouvement/ } \\
\text { menaces d'autres personnes) } \\
\text { Qualité des relations avec autrui } \\
\text { Présence ou absence de public et son expressivité } \\
\text { La manière de voir, entendre autrui } \\
\text { Modalité de déplacement } \\
\text { Appropriation de l'espace }\end{array}$ \\
\hline
\end{tabular}

Fig. 6 Grille d'analyse sensorielle [28] 


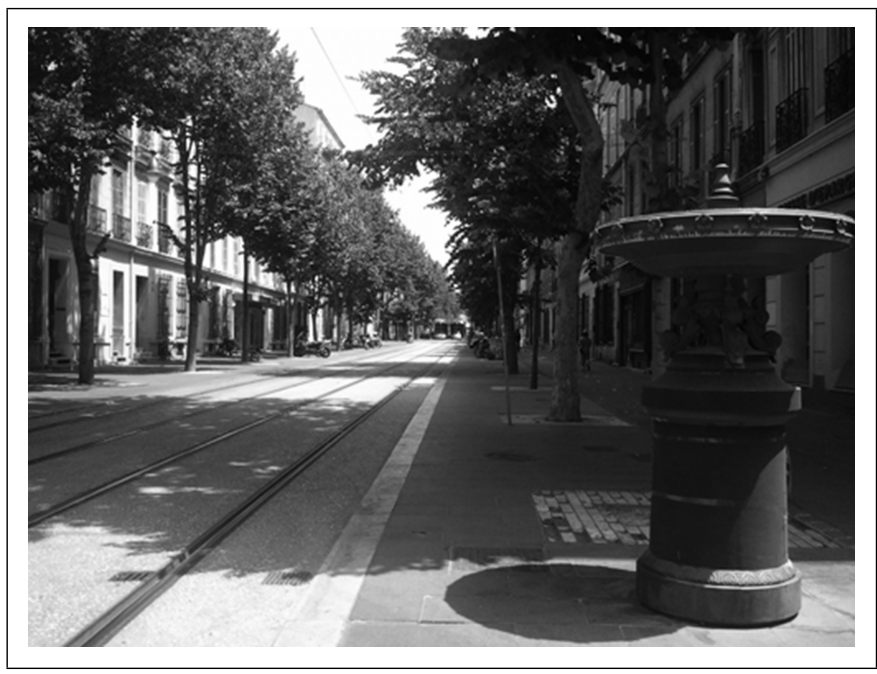

Fig. 7 Le boulevard Longchamp [28]

et la perspective sur le palais Longchamp confèrent une qualité paysagère remarquable à cette séquence. Par ailleurs, l'analyse en termes de lisibilité met en évidence que chaque extrémité de la séquence, les Réformés et le palais Longchamp, est clairement identifiée. La vision de ces points de repères majeurs apporte de l'unité à la séquence. Les stations de tramway constituent des points de repère ponctuels. La position des stations au niveau du carrefour renforce leurs visibilités et permet aux piétons d'organiser par étape leur image des aménagements de tramway.

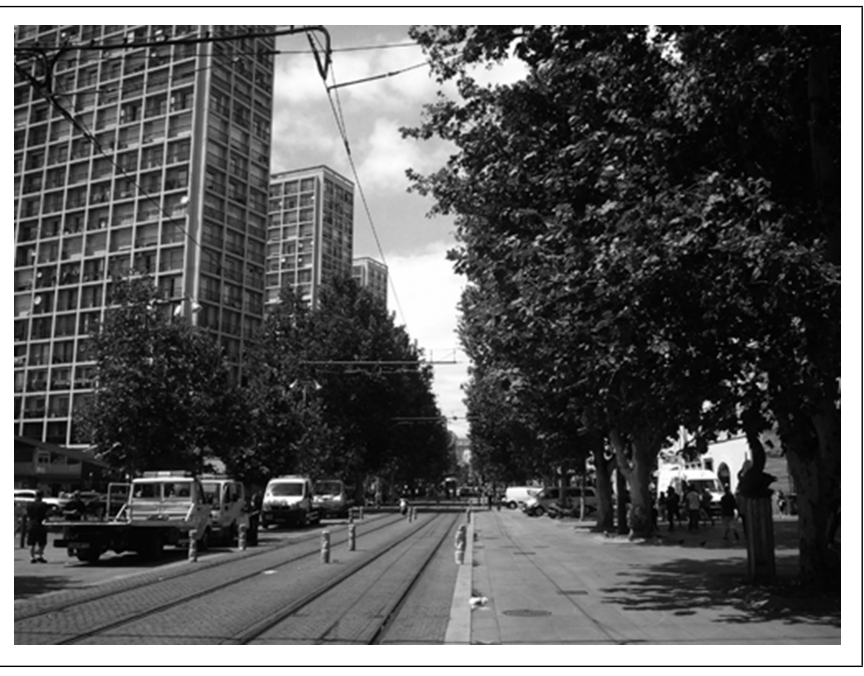

Fig. 9 Cours Belsunce [28]

Le cours Belsunce évoque « un cours urbain » dans le sens où il constitue une aire piétonne attractive, avec une largeur de voie apportant un effet d'aération.

Le milieu ambiant de la séquence du cours Belsunce est « saturé » par la diversité d'usages et de population, et devient «ambigu » au niveau de la traversée. En effet, la luminosité vive de cette séquence est troublante, elle semble éteindre les couleurs et estompe toute démarcation physique. Les seuils et limites de l'espace public ne sont pas clairement perçus par les piétons. Au niveau sonore, un bourdonnement permanent dû à la foule de piétons, se

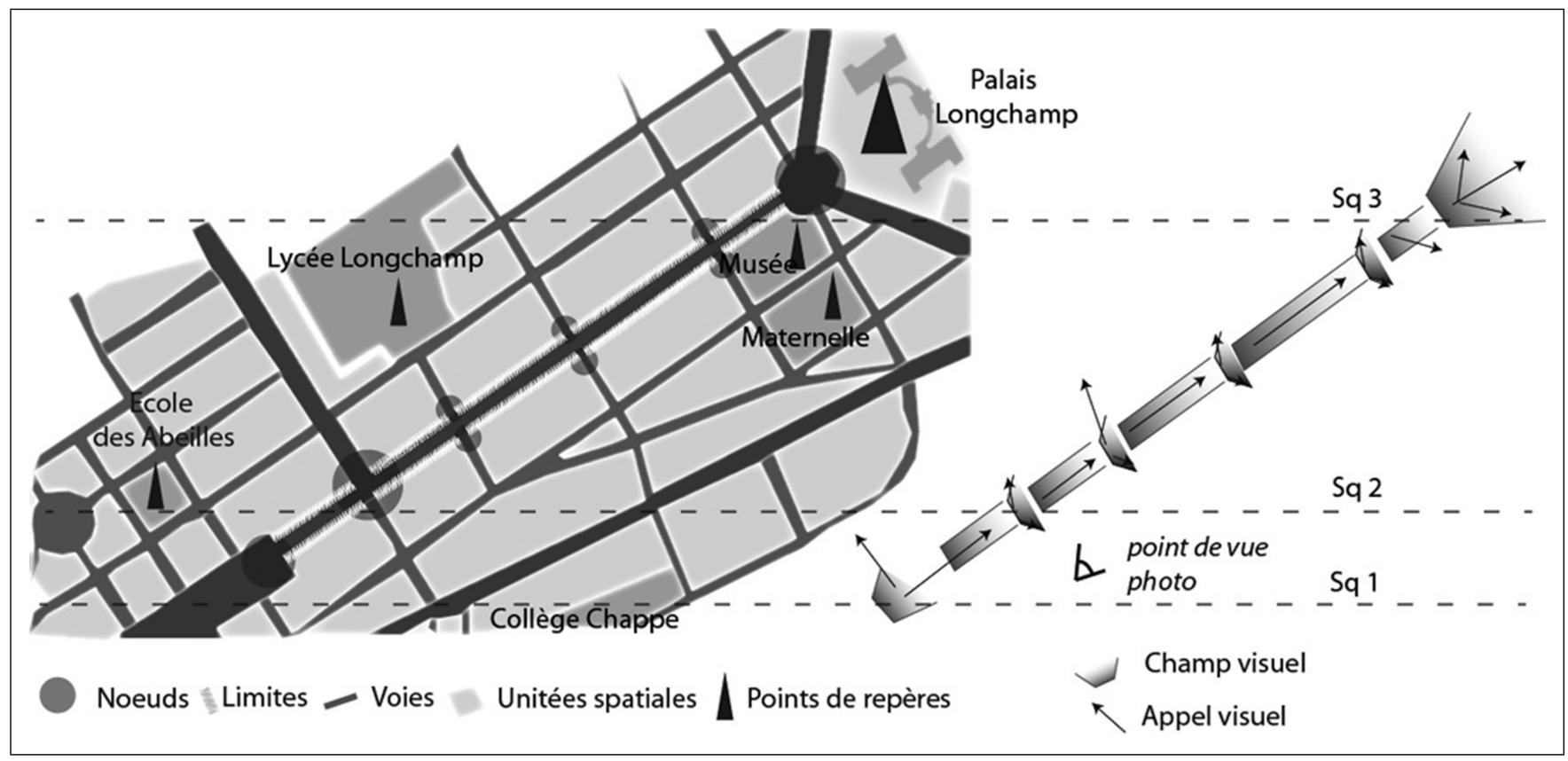

Fig. 8 Éléments de lisibilité de la séquence du boulevard Longchamp [28] 


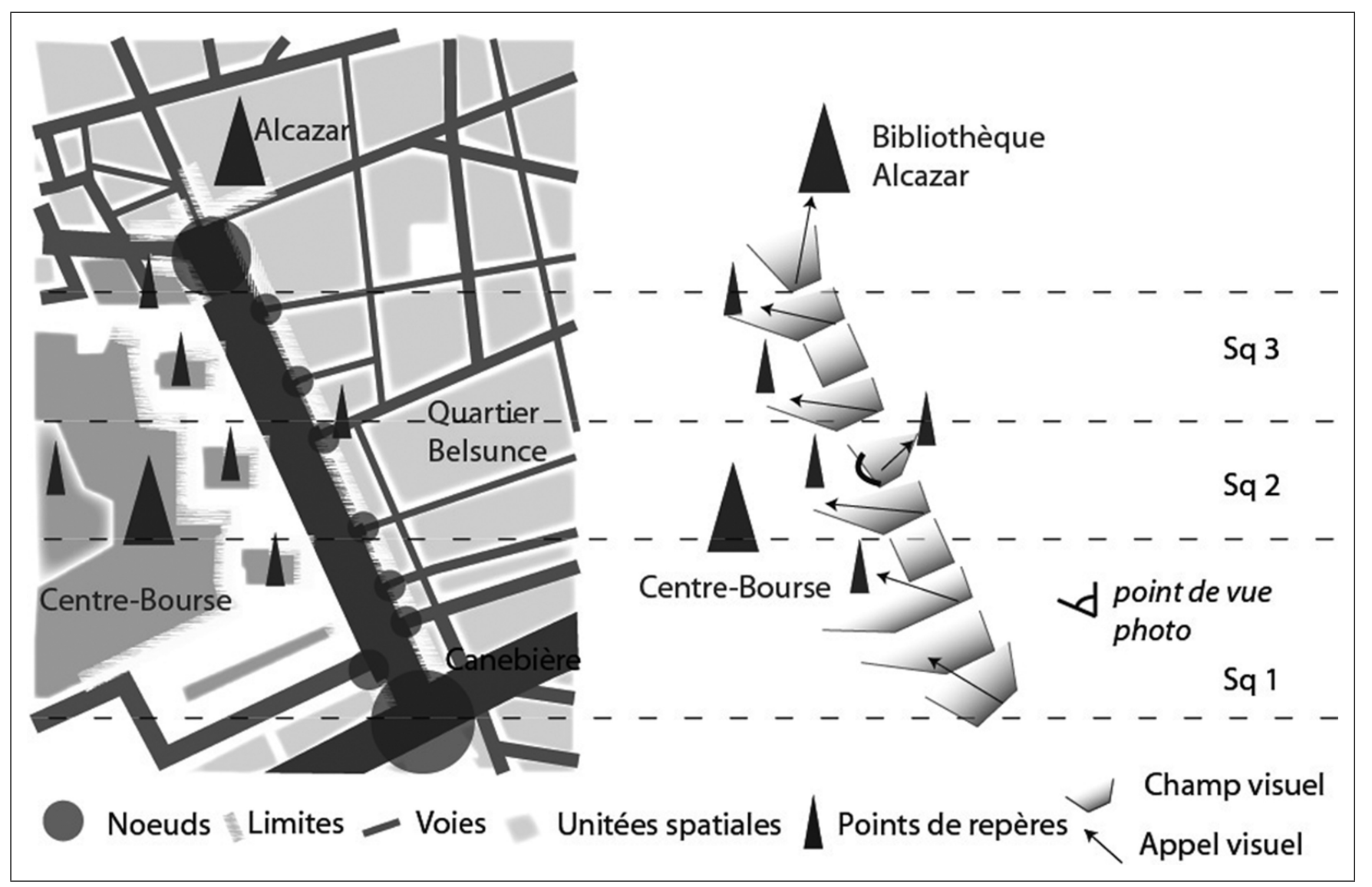

Fig. 10 Éléments de lisibilité de la séquence du cours Belsunce [28]

mêle à une rumeur mécanique des circulations. Concernant les éléments de lisibilité, la position d'éléments connus tels que le Centre Bourse d'un côté et l'aire piétonne de l'autre côté de la voie met en évidence des qualités directionnelles. Seule la présence de l'alignement de hauts platanes semble canaliser le contraste et l'asymétrie du lieu. En effet, un fort contraste s'opère (notamment de hauteur et de typologie bâtie) entre les tours d'habitat collectif de dix-huit étages et les hôtels particuliers de type Haussmannien à quatre étages. De plus, l'unité spatiale du Centre Bourse peut être qualifiée d'extravertie de par sa connection au reste de la ville, tandis que celle du quartier Belsunce semble introvertie. Enfin, l'absence d'alignement à la voie, la discontinuité du bâti, les stationnements anarchiques, les circulations motorisées non autorisées sur la plateforme de tramway, sont des éléments pas facilement assimilables et rendent les aménagements de tramway peu lisibles pour les piétons.

La Canebière évoque un «axe structurant symbolique ». Le milieu ambiant de la séquence de la Canebière peut être qualifié $d^{\prime}$ ' " attractif » mais devient davantage « saturé » par la diversité de flux qui convergent. En effet, la perspective sur le Vieux Port, la qualité architecturale des

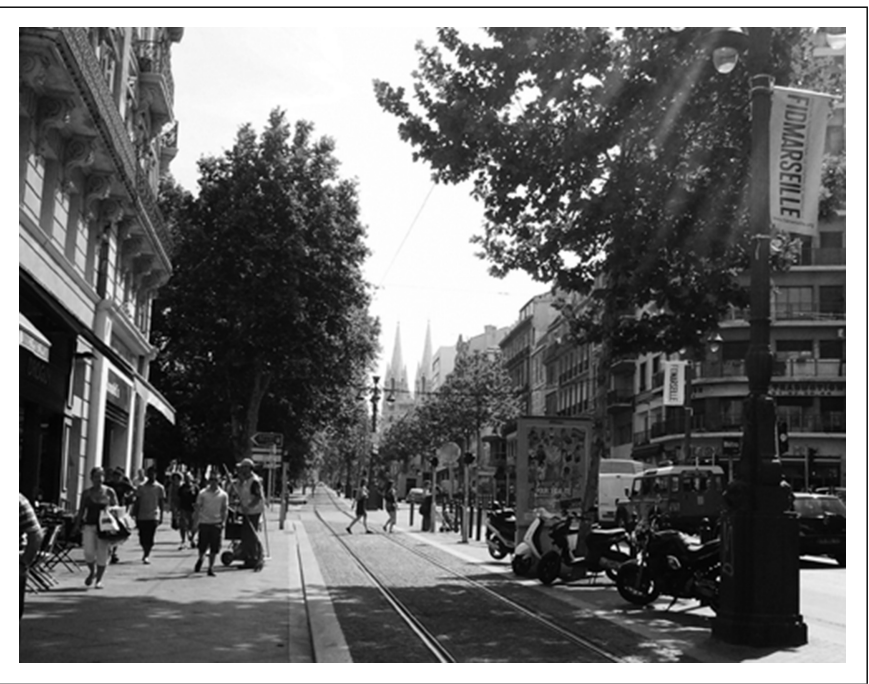

Fig. 11 La Canebière [28]

façades, la promenade sur les larges trottoirs, les achats dans les divers commerces et échoppes alimentaires constituent l'essence même du lieu. Chacune de ses caractéristiques renforce l'identification de la séquence et la facilité des 


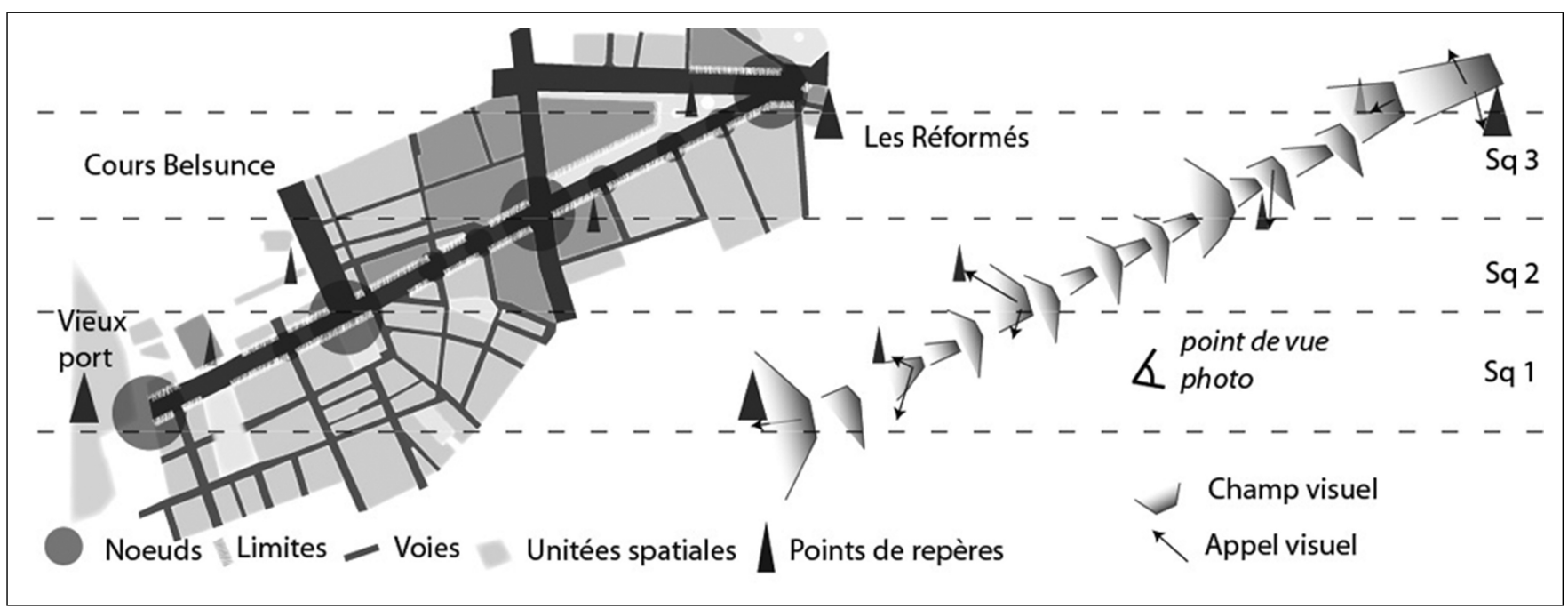

Fig. 12 Éléments de lisibilité de la séquence de la Canebière [28]

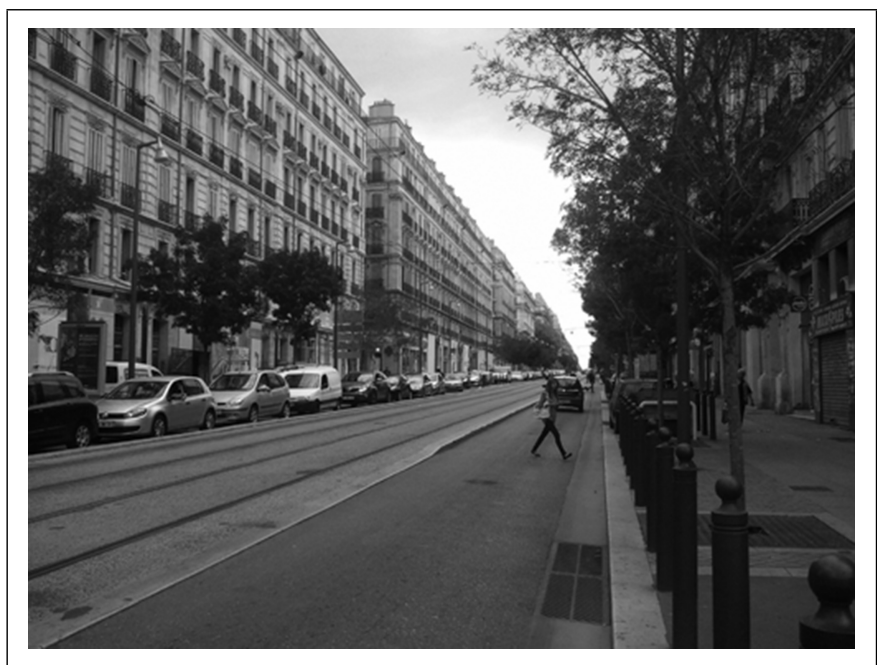

Fig. 13 Rue de la République [28]

piétons à s'orienter. De plus, la Canebière est un nom associé au centre-ville de Marseille. La symbolique de cette séquence réside ainsi dans l'association du nom de la voie et de la désignation du centre-ville. Cependant, la Canebière, en tant que symbole du centre-ville de la commune, lieu de rencontre, d'allégresse et de convivialité tend à s'éloigner de son «génie du lieu » [34]. Cette séquence, saturée par les flux de circulation, provoque des coupures, des nuisances sonores lors du parcours des piétons. Des problèmes de lisibilité des aménagements de tramway se posent pour les piétons et complexifient leur perception lors de la traversée.

La rue de la République évoque « un axe urbain structurant minéral ».
Le milieu ambiant de la séquence de la rue de la République peut être qualifié de «transitoire ». En effet, les usagers de cette séquence semblent ne faire que passer pour atteindre une autre destination. La luminosité vive et la faible présence végétale accentuent une minéralité $\mathrm{du}$ lieu peu accueillante pour l'usager piéton. De plus, la continuité du bâti et son parfait alignement à la voie mettent en évidence un effet de visée invitant la poursuite du parcours des piétons vers le Vieux Port. Cette incitation au déplacement des piétons vers le Vieux Port s'accentue par le contraste qui s'opère au niveau de la place Sadi-Carnot, entre la partie Joliette (qui comporte de nombreux locaux fermés, des commerces de type bazar ou alimentaires de proximité) et la partie Vieux Port (davantage représentative d'un hypercentre de métropole). Au niveau sonore, la rumeur mécanique des nombreux travaux est assourdissante. De plus, des odeurs de poussière et de pollution sont particulièrement désagréables pour les piétons. Concernant les éléments de lisibilité, la structure de la voie accentue un effet de percée du tissu urbain. Les unités spatiales sont clairement identifiables par leurs caractéristiques physiques de type Haussmannien et elles contrastent avec le reste de la ville, notamment avec le quartier du Panier constitué de ruelles tortueuses de type médiéval. Des brèches de lisibilité persistent cependant au niveau des liaisons avec les autres voies en intersection.

La méthode employée construit un outil d'évaluation des milieux ambiants. Cette méthode caractérise les environnements urbains des espaces publics aménagés de tramway. Ces derniers mettent en scène un ensemble de ressources et de contraintes pour les piétons, variant à la fois selon la fonctionnalité de l'aménagement viaire mais aussi selon l'atmosphère particulière, la physionomie et les usages du lieu. Après avoir abordé l'échelle de la séquence, nous 


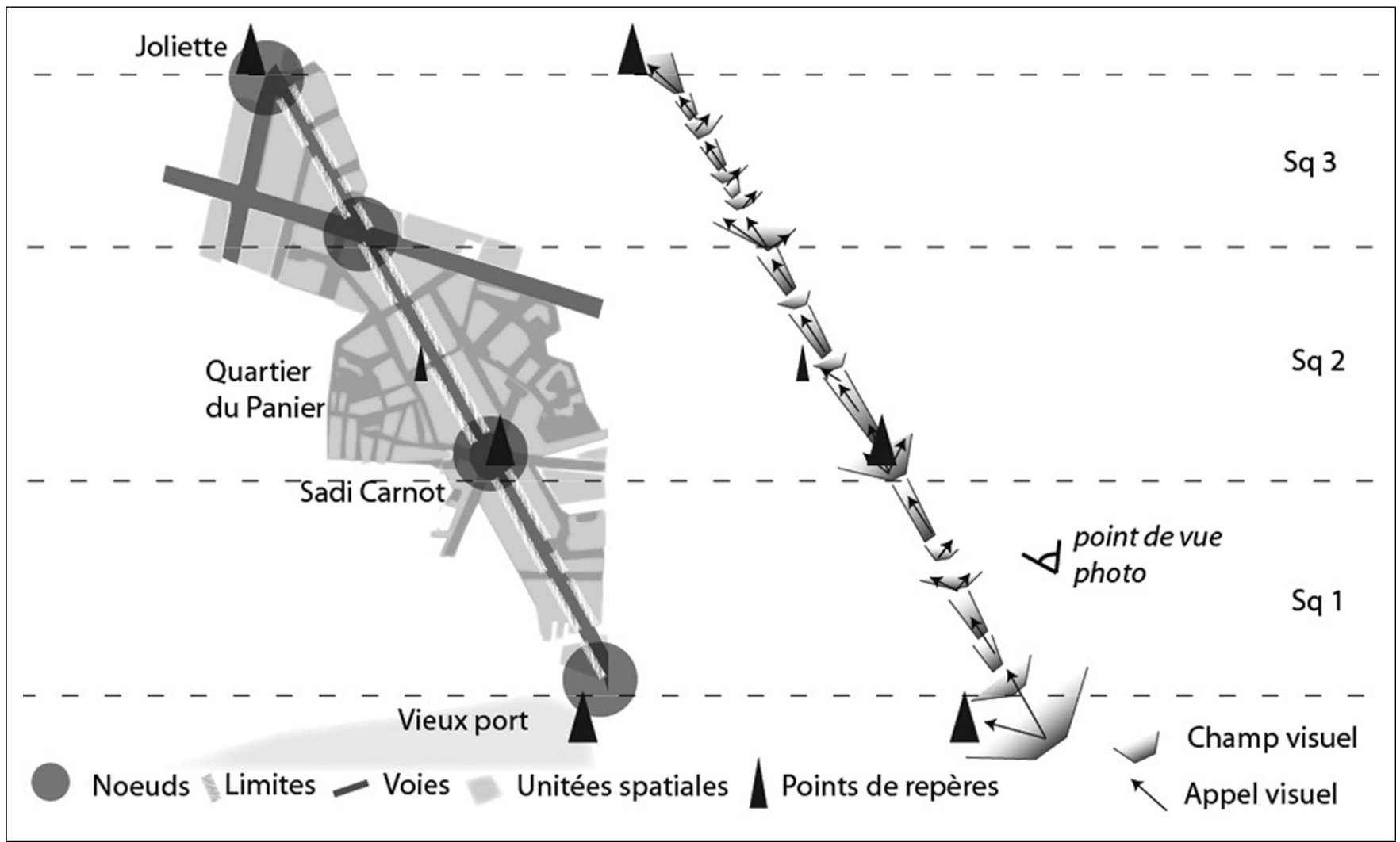

Fig. 14 Éléments de lisibilité de la séquence de la rue de la République [28]

analyserons plus spécifiquement la diversité des pratiques piétonnes à l'échelle de la traversée des aménagements de tramway.

\section{À l'échelle de la traversée piétonne : des comportements et des usages}

À l'échelle plus fine de la traversée des aménagements de tramway, les comportements et les perceptions des piétons sont analysés pour chacune des séquences spécifiques. En prenant en compte la problématique des déplacements piétons dans un environnement construit, cette approche a pour objectif de faire émerger des tendances de comportements collectifs à partir de comportements individuels de piétons.

\section{La plongée dans l'univers des pratiques}

La plongée dans l'univers des pratiques permet de recueillir et d'analyser précisément les comportements et les perceptions des piétons lors de la traversée de voie de tramway. Les piétons sont ainsi observés directement sur le terrain, pour évaluer et noter de manière rigoureuse leurs comportements lors de leur traversée. Les informations recueillies sont précises et l'échantillon de piétons pour chacune des quatre séquences se veut le plus représentatif possible de la population de la commune. La technique la plus rapide et retranscrivant de la manière la plus juste les observations des comportements des piétons en situation de traversée, est celle des croquis annotés (Fig. 15). Ainsi, un codage des sémiologies utilisées est effectué et permet de représenter graphiquement chaque traversée des piétons observés.

Le compte rendu des trajectoires, regards, rythmes des piétons permet de conserver une trace matérielle de leur activité in situ, de pouvoir les consulter de nombreuses fois, de les analyser, tout en les contextualisant avec l'environnement urbain alentour. De retour du terrain d'étude, un travail de recueil et d'analyse des données est effectué. À la suite de la réalisation de cartes d'analyse thématique, deux fiches synthétiques sont réalisées afin de comparer les séquences entre elles. Enfin, des combinaisons de regards et rythmes sont réalisées en fonction des trajectoires et permettent de suivre l'évolution du comportement de chaque piéton pour toutes les étapes de traversée des aménagements de tramway. Un découpage est ainsi défini distinguant les zones d'approches comme le trottoir et les zones proprement dites de traversée, comme la plateforme tramway ou la voie routière [7].

Par la suite, la méthodologie utilisée met en corrélation les observations des pratiques dans leurs contextes, 


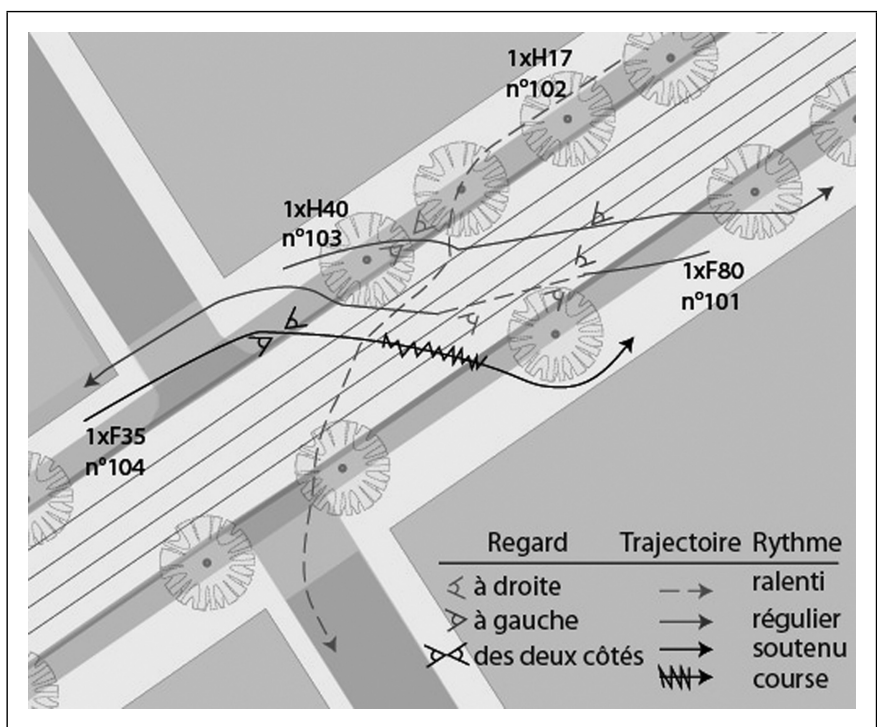

Fig. 15 Modèle d'annotations des observations sur le terrain d'étude [28]

avec les perceptions des piétons. En effet, l'objectif est d'appréhender les comportements au cours de la traversée et d'obtenir des informations sur les perceptions des piétons. Les piétons interrogés sont ceux qui ont été observés lors de la traversée. La grille du questionnaire a été élaborée pour apporter des informations sur la perception des piétons de leur traversée en termes de sûreté, de facilité, d'agrément, de rapport de priorité, de conception de la marche, de relation avec les autres usagers et de rapport à la règle. De plus, cette méthode met en évidence les éléments nécessitant particulièrement l'attention des piétons, leur fréquence de traversée et l'origine puis la destination de leur déplacement. Enfin, est considérée l'évocation personnelle de chaque piéton concernant la séquence, les principaux éléments identifiés et les suggestions d'améliorations de la traversée. Certaines données sont ensuite confrontées et croisées entre elles. Ainsi, l'insécurité perçue par les piétons lors de leur traversée est croisée avec les éléments nécessitant l'attention. La difficulté perçue par les piétons lors de leur traversée est mise en corrélation avec les améliorations qu'ils considèrent nécessaire d'apporter. Enfin, les désagréments perçus par les piétons sont mis en lien avec ce que la séquence leur évoque.

\section{Des identités de piéton}

Au niveau du terrain d'étude, 50 observations et 50 questionnaires ont été réalisés sur chacune des quatre séquences définies précédemment avec des piétons venant d'effectuer la traversée. Le choix des piétons observés et interviewés correspond à un échantillonnage préétabli et représentatif de la population de la commune. Les piétons enquêtés ne tirent pas de valise et n'ont pas de poussette car ces derniers constituent des cas d'études spécifiques en terme de perception et de mobilité. Les séquences sont comparées deux à deux, en commençant par les deux aires piétonnes.

Concernant la traversée du boulevard Longchamp, les trajectoires des piétons s'étalent en longueur : les piétons s'approprient la plateforme de tramway, ils s'y attardent, investissent l'espace, ils sont en position d'échange avec autrui. Les piétons adoptent un rythme souple, plutôt ralenti. La lenteur du rythme signifie "la volonté de ne pas brusquer le temps, de ne pas se laisser bousculer par lui », "d'augmenter notre capacité d'accueillir le monde et de ne pas nous oublier en chemin » [35]. Par ailleurs, les piétons regardent en majorité tout droit et effectuent peu de vérification de la circulation.

S'agissant de la traversée du cours Belsunce, les trajectoires sont éparpillées et directes. Les piétons traversent sans rupture de rythme et centrent leur attention sur leur déplacement. Ils mobilisent leur appareil perceptif pour anticiper les événements de leur environnement urbain avant la traversée. Puis ils regardent des deux côtés et à deux reprises durant leur traversée. Les piétons sont en situation de paradoxe : à leur place au sein de cette aire piétonne attractive, ils sont en position incongrue lors de la traversée. La circulation non autorisée d'automobilistes, cyclomotoristes, cyclistes sur la plateforme de tramway rend la traversée conflictuelle pour les piétons. De plus, les difficultés à percevoir les bornes de leur environnement urbain et leur exposition au champ de vision d'autrui placent les piétons dans une situation d'étranger. Au sein de cette séquence le piéton est, comme le mentionne Simmel [36], « le voisin des membres du groupe social, puisqu'il cohabite avec eux, et pourtant il est la figure de ce qui est extérieur au groupe ».

Sur ces deux aires piétonnes traversées par une plateforme tramway, des divergences de qualifications d'ambiances sont apparues et entrainent des pratiques différentes. Les perceptions des piétons confirment ces observations. En effet, sur le cours Belsunce, les piétons se sentent moins à l'aise en traversée que sur le boulevard Longchamp : ils la jugent difficile à $14 \%$ contre $0 \%$ sur Longchamp, ils disent ne pas se sentir à l'aise (38\% contre $18 \%$ ), ils évoquent des "gênes sonores », la « saleté », les « circulations anarchiques », les « incivilités» et le « désordre ». Cette intrusion de la circulation motorisée sur le cours Belsunce réapparaît au travers de différentes réponses des piétons (conseils pour traverser, suggestion d'amélioration...). Elle explique bien le caractère conflictuel de la traversée. À l'inverse, sur le boulevard Longchamp, les piétons trouvent la traversée agréable (94\% contre $20 \%$ sur Belsunce). Ils s'y sentent à l'aise, au point que celle-ci est la traversée où ils ont le moins conscience de la priorité du tramway. 
La majorité des piétons interrogés étaient des coutumiers (tous fréquentent le lieu au moins une fois par semaine). La comparaison des perceptions et des pratiques met en lumière l'effet de l'ambiance sur les cheminements des piétons. Pour les aménageurs, elle incite à plus d'attention quant aux espaces « privilégiés » et «attractifs » pour les piétons car ces derniers peuvent les entraîner à baisser leur vigilance vis-à-vis des véhicules y compris le tramway. En particulier, les aires piétonnes agréables et apaisées de tout trafic en dehors du tramway incitent peu les piétons à rester vigilants par rapport à l'arrivée éventuelle d'un tramway.

Les sites de la Canebière et de la rue de la République sont composés de trois parties de traversée (Fig. 5), avec soit la plateforme tramway en premier puis en dernier (bilatérale) et la voie routière en axiale, soit l'inverse. Ils sont donc soit confrontés d'abord à la plateforme soit à la voie routière. Les ambiances diffèrent et renvoient à des comportements distincts.

Dans la traversée de la Canebière, les piétons piétinent, leur trajectoire est déviée par des obstacles, ils sont ainsi contraints de se détourner de leur trajectoire en utilisant la zone refuge, et leur rythme est impatient. Les piétons développent une attention distribuée et effectuent des vérifications en plusieurs temps. Les piétons ont tendance, de manière générale, à anticiper le trafic des voies de circulation automobile situées en axiale dès le trottoir. Le faible trafic de la plateforme permet aux piétons d'étendre leur champ de vision.

Dans le cas de la traversée de la rue de la République, les trajectoires s'effectuent en diagonales directes. Les piétons séjournent peu et échangent peu, ils ont tendance à privilégier le plus pratique. Les piétons ne font que passer, leur traversée est habituelle et ils maintiennent une attention centrée. Ils concentrent leur attention sur la première voie de circulation automobile et n'anticipent pas le reste de la traversée. Ils reprennent ainsi de l'information en lançant des regards des deux côtés au niveau de la plateforme en situation axiale.

$\mathrm{Au}$ niveau des interviews, les piétons traversant la séquence de la Canebière perçoivent davantage de danger et d'insécurité que ceux traversant la rue de la République. Sur la Canebière, ils conseillent d' " être vigilant » et d' « emprunter les passages piétons », ils suggèrent de « supprimer la circulation automobile pour mieux repérer et entendre les tramways ». En fait, cette insécurité relève davantage de la circulation automobile et notamment de ses désagréments comme les « embouteillages », «la cacophonie totale », la «pagaille ».

Concernant la rue de la République on ne retrouve pas les appels à supprimer les circulations automobiles que l'on enregistre sur la Canebière. Sur la rue de la République, les piétons évoquent davantage le besoin de faire attention aux passages des tramways. Ils sont plus conscients d'avoir traversé une plateforme tramway que sur le Canebière. La comparaison des deux sites tend à montrer que le tramway est mieux perçu par les piétons lorsque la plateforme est en position axiale et que les deux sens de circulation sont regroupés. Quand la plateforme est unilatérale, elle est vue comme le prolongement du trottoir.

D'une manière générale, la comparaison des deux sites montre que la circulation automobile, lorsqu'elle est présente, reste un critère important de perception pour les piétons. Si les trafics différents entre voies routières et plateforme tramway peuvent expliquer cela, la dangerosité du tramway et son caractère prioritaire auraient pu contrecarrer cet effet. Apparemment il n'en est rien et même sur les sites à faible trafic routier, la prégnance des «voitures » reste plus forte que le passage des tramways. Les deux tronçons étudiés (Canebière et République) sont caractérisés par des ambiances et des niveaux de circulation assez distincts, comme l'a montré l'analyse des séquences, ce qui entraîne une difficulté si l'on veut pousser plus loin la comparaison.

Sur les quatre séquences étudiées, la définition des milieux ambiants croisée avec les différentes tendances comportementales a permis d'affiner la connaissance des pratiques et usages des piétons au sein des espaces publics aménagés de tramway. L'environnement sensible des aménagements de tramway s'est révélé important à considérer car il influence grandement sur les déplacements et l'attention des piétons, en particulier lorsque la plateforme est située en aire piétonne. Au niveau des séquences comportant une traversée plus complexe, le trafic motorisé a tendance à davantage influencer le comportement des piétons. Cette étude permet ainsi d'appréhender la complexité de la logique des piétons en déplacement. Cette méthode construite sur différentes échelles et appliquée sur une diversité d'espaces publics aménagés de tramway, auquel les piétons peuvent être confrontés, permet d'avancer sur la question de la compréhension piétonne des aménagements de tramway. Afin d'approfondir davantage les résultats, une étude similaire nécessiterait d'être réalisée sur un nombre plus important de sites, présentant le moins possible de différences de trafic motorisé.

\section{Conclusion}

Ce travail s'intéressait à la question de la perception par les piétons des aménagements de tramway pour mieux déterminer les conflits potentiels. La mise en place d'une méthode adaptée s'est vite révélée nécessaire dans la mesure où de nombreux éléments sont à prendre en compte dans une vision globale. C'est ainsi qu'ont été mobilisés à la fois des éléments d'ordre physique (morphologie urbaine), social (relation entre les individus, usages) et sensible 
(ambiances). L'originalité de la méthode proposée est également le jeu des échelles : elle établit un diagnostic de l'ensemble de la ligne de tramway et plonge dans l'univers des pratiques piétonnes de la traversée de la plateforme. En effet, la prise en compte du contexte et du milieu ambiant de l'environnement urbain d'un tronçon de tramway est primordiale avant de s'intéresser plus spécifiquement à l'analyse de la traversée piétonne. Cette imbrication d'échelles permet de reconsidérer l'espace public aménagé de tramway dans son ensemble. L'analyse a ainsi fait apparaître l'importance des ambiances, de la qualité paysagère, de la composition urbaine et des usages dans la prise en compte de la perception des piétons. Enfin, ce travail - en permettant de mieux comprendre les stratégies de déplacement des piétons, en fonction des contraintes, des nuisances ou opportunités de l'environnement urbain mouvant des espaces publics aménagés de tramway -, permet d'apporter des éléments de réflexion pour la planification urbaine.

\section{Références}

1. Harman R, Menerault P, L'Hostis A (2007) Public transport in cities and regions, Facing an uncertain future?. In: Booth $\mathrm{P}$, Breuillard M, Fraser C, Paris D (2007) Spatial Planning Systems of Britain and France, A comparative analysis. Routledge, pp 188-205.

2. Hamman $\mathrm{P}$ (2011) Le tramway dans la ville, le projet urbain négocié à l'aune des déplacements. Presses universitaires de Rennes, coll. « Espace et territoires », Rennes, pp 73-89.

3. Frenay $P$ (2005) Le tram, outil en faveur d'une ville durable? Réflexions tirées d'une comparaison entre quelques villes moyennes françaises et alémaniques. Transport, environnement, circulation 185: 2-8.

4. Lynch K (1999) L'image de la Cité, trad. par Vénard M-F et Vénard J-L de The Image of the City (1960). Dunod, Paris, $221 \mathrm{p}$.

5. Huguenin-Richard F (2003) Comportements, tactiques et conduites déviantes de piétons en situation de traversée complexe : le cas du franchissement de voies en site propre dans Paris. Ed Laboratoire ENEC, pp 91-107.

6. Maestracci M (2010) Analyser les comportements pour lutter contre l'insécurité des déplacements piétons. Proceedings: Congrès ATEC-ITS France, 3 et 4 février 2010, Versailles, 13 p.

7. Millot M (2012) Traversée piétonne des sites tramways : quels comportements des piétons ? in Granié M-A, Auberlet J-M, Dommes A, Serre T (2012) Qualité et sécurité du déplacement piéton : facteurs, enjeux et nouvelles actions. Les collections de l'INRETS, pp 185-195.

8. Hedelin A, Björnstiget U, Brismar B (1996) Tram-A risk factor for pedestrians. Accident Analysis and Prevention 28(6): 733-738.

9. Cameron IC, Harris NJ, Kehoe NJS (2001) Tram-related injury in Sheffield. International Journal of the Care of the Injured 32: 275-277.

10. Beer S, Brenac T (2006) Tramway et sécurité routière, l'expérience des pays germanophones. Transport, environnement, circulation 190: 40-47.

11. ISBR (2009) Etude des accidents entre un tramway et un piéton en région de Bruxelles-capitale de 2004 à 2006. 71 p.
12. Currie G, Tivendale K, Scott R (2011) Analysis and mitigation of safety issues at curbside tram stops. Transportation Research Record 2219: 20-29.

13. Maes P (1989) The dynamics of action selection, In proceedings of International Joint conference on Artificial Intelligence (IJCAI-89), Detroit, Michigan USA.

14. Granié M-A, Auberlet J-M (2008) Le piéton et son environnement. Quelles interactions? Quelles adaptations? Actes INRETS 115: 95105 .

15. Merlin P, Choay F (année ?) Dictionnaire de l'urbanisme et de l'aménagement. Revu et augmentée, PUF, coll. «Quadrige dicos poche $»$, Paris.

16. Chelkoff G, Thibaud J-P (1993) L'espace public, modes sensibles. Les annales de la recherche urbaine : 57-58: 6-16.

17. Augoyard J-F (1979) Pas à pas. Essai sur les cheminements quotidiens en milieu urbain. Le Seuil, Coll. « Espacements », Paris, $185 \mathrm{p}$.

18. Goffman E (1974) Les rites d'interaction, Minuit, Paris, pp 43-85.

19. Le Breton D (2000) Marche urbaine in Eloge de la marche. Métaillé, Paris, pp 121-146.

20. Thomas R (2005) Les trajectoires de l'accessibilité. À la croisée, coll. « Ambiances, ambiance », $183 \mathrm{p}$.

21. Chelkoff G (1997) Formes, formants et formalités. L'espace urbain en méthodes, pp 101-127.

22. Ewing R, Handy S, Brownson R-C, Clemente O, Winston E (2006) Identifying and measuring urban design qualities related to walkability. Journal of Physical Activity and Health 3: 223-240.

23. Himanen V, Kulmala R (1988) An application of logit models in analysing the behaviour of pedestrians and car drivers on pedestrian crossings. Accident Analysis \& Prevention 20: 187-197.

24. CETUR (1983) Sécurité des piétons lors de leur traversée de chaussées. Les dossiers du CETUR 21, Centre d'Études des Transports Urbains, France, $112 \mathrm{p}$.

25. Granié M-A, Auberlet J-M (2008) Le piéton et son environnement. Quelles interactions? Quelles adaptations? Actes INRETS 115: 163-174.

26. Stransky V(2011) Une méthode d'analyse spatiale des espaces piétons au service d'un urbanisme orienté vers le rail. Recherche Transport Sécurité 27: 178-199.

27. Grosjean M, Thibaud J-P (2001) L'espace urbain en méthodes. Parenthèses, Coll. «Eupalinos », Marseille, 214 p.

28. Maitre E (2012) Le piéton et son environnement urbain : clés de lecture piétonne des environnements de tramway. Mémoire de fin d'étude, Institut d'Urbanisme et d'Aménagement Régional d'Aixen-Provence, $178 \mathrm{p}$.

29. Lévy E (2001) Saisir l'accessibilité : les trajets-voyageurs à la gare du Nord. In: Grosjean M, Thibaud J-P L'espace urbain en méthodes. Parenthèses, Marseille, pp 47-62.

30. De Certeau M (1980) L'invention du quotidien, Arts de faire. Galimard, 349 p.

31. Hankin B-D, Wright R-A (1958) Passenger flow in subways. Operational Research Quaterly 9(2): 81-88.

32. Adolph L (1998) In : Ambiance architecturale et urbaine. Les cahiers de la recherche architecturale 42/43: 7-12.

33. Coulter J, Parsons E-D (1990) The praxiology of perception: visual orientations and pratical action. Inquiry. An interdisciplinary journal of philosophy 33(3): 251-272.

34. Norberg Schulz C (1997) Genius Loci, paysage, ambiance, architecture. Mardaga, pp 5-202.

35. Sansot $\mathrm{P}$ (1998) Du bon usage de la lenteur. Petite Bibliothèque, coll. « Rivages Poche », n³13, 65 p.

36. Simmel G (1981) Préface de Freund J, Sociologie et Épistémologie, PUF, Paris, $58 \mathrm{p}$. 